\title{
Impulsividad y agresividad en cadetes de policía
}

\section{Impulsiveness and Aggressiveness in Police Cadets}

\author{
Horacio Daniel García ${ }^{1}$ \\ Universidad Nacional de San Luis, Facultad de Psicología, Argentina
}

Resumen. El objetivo principal fue indagar los niveles de agresividad e impulsividad en Cadetes de Policía de San Luis, Argentina. La muestra fue intencional, no probabilística, conformada por 58 alumnos (39 varones y 19 mujeres) que cursan el segundo y tercer año de un instituto de formación. Fueron administrados dos instrumentos: Cuestionario de Agresividad (AQ) (Andreu-Rodríguez, Peña-Fernández \& Graña-Gómez, 2002) y la Escala de Impulsividad de Barratt (BIS-11) (Oquendo et al., 2001). Las principales conclusiones evidencian niveles elevados de agresión física e impulsividad cognitiva. Las mujeres mostraron puntuaciones elevadas en la subescala hostilidad, mientras que los hombres mayores niveles de agresión física; los alumnos del último año de formación exhibieron niveles elevados de impulsividad motora, agresión verbal e ira. Finalmente, se corroboraron asociaciones positivas entre las variables de impulsividad y agresividad.

Palabras clave. Cadetes de policía, agresividad, impulsividad.

Abstract. The main objective was to inquire the levels of aggressiveness and impulsivity in Police Cadets of the San Luis, Argentina. The sample was intentional, not probabilistic, and consisted of 58 students (39 males and 19 females) attending the second and third year of a training institute. Two instruments were administered: The Aggressiveness Questionnaire (AQ) (Andreu-Rodríguez, Peña-Fernández \& Graña-Gómez, 2002) and the Barratt Impulsivity Scale (BIS-11) (Oquendo et al., 2001). The main conclusions make refer to high levels of Physical Aggression and Cognitive Impulsiveness. Women showed high scores on the Hostility sub-scale, while men had higher levels of Physical Aggression; the students of the last year of training exhibited elevated levels of Motor Impulsiveness, Verbal Aggression and Wrath. Finally, positive associations between the variables of Impulsivity and Aggressiveness to were corroborated.

Keywords. Police cadets, aggressiveness, impulsiveness.

${ }^{1}$ Horacio Daniel García. Universidad Nacional de San Luis, Argentina. Dirección Postal: Pringles 1356, dpto. 10, San Luis, San Luis Capital, Argentina. CP 5700. E-mail: hdgarcia69@gmail.com 


\section{Introducción}

En los últimos años la institución policial se ha visto interpelada por la sociedad en la medida en que los medios masivos de comunicación refieren a diario hechos de corrupción, abuso de la autoridad, excesos en la represión, entre otros tópicos relacionados con esta entidad. Numerosas investigaciones se han abocado a analizar el accionar concreto de la actuación policial y su relación con la comunidad, exponiendo la necesidad de maximizar la formación (académica y profesional) de los funcionarios encargados de mantener el orden público, la profesionalización del personal, la descentralización jerárquica de quienes la conforman y su adhesión a los derechos humanos. Así el panorama, la presente investigación se centra en el estudio de algunas variables psicológicas de incidencia directa en las futuras prácticas laborales de los integrantes de la muestra, en tanto constructos psicológicos complejos con serias implicaciones sociales en temas como la violencia, la conducta de riesgo y la adaptación social (Folino, Escobar-Córdoba, \& Castillo, 2006).

\section{La institución policial en Argentina: breve referencia}

En Argentina, cada uno de los gobiernos provinciales cuenta con un elevado grado de autonomía para determinar la estructura de sus fuerzas policiales, su dependencia institucional y la modalidad de formación que se impartirá a sus miembros. Lo anterior ha derivado en una amplia heterogeneidad en la formación de los recursos humanos que integran los cuerpos policiales y las fuerzas de seguridad (Alonso, 2008).

Tudela-Poblete (2010) afirmó que "cada institución policial es una cultura organizacional" (p. 78). Esto es “... un paradigma cognitivo para los miembros de la organización, en tanto describe, explica, prescribe y consolida una visión del mundo, funda y recrea actitudes, valores, normas y creencias, conductas, entre otros componentes" (p. 77), los cuales son tácitamente aceptados por los agentes policiales y requieren ser internalizados por los nuevos miembros.

La lucha contra el delito, la presencia constante de peligro, el uso de la violencia y su consecuente autorización a emplear la fuerza física, remiten a elementos relativos a la naturaleza y las circunstancias en que el policía realiza su tarea. Estos factores constituirían una serie de rasgos definitorios de la labor policial y confluirían en la construcción de una “... personalidad, estilo o carácter del trabajo policial, no como un fenómeno individual, sino como una matriz socialmente construida y transmitida mediante procesos de socialización" (Tudela-Poblete, 2010, p. 81).

Impulsividad y agresividad

La violencia y la agresión han sido objeto de interés desde los orígenes de la psicología, aunque recién en el siglo XX se convirtieron en un tema de interés científico. Etimológicamente, la palabra agresividad, proviene del latín aggredio, que se descompone en los radicales ad gradi (gradus = "paso" y ad = "hacia"). Significa avanzar, dar un paso hacia delante, acometer a otro. Probablemente "agredir" adquirió el sentido de atacar al comprobarse que en la guerra el avance siempre solía ser el inicio de un ataque o contienda (Fromm, 1986). "Ser agresivo, de acuerdo con el sentido original, es pues avanzar hacia un objetivo, sin vacilación indebida, duda ni temor" (Fromm, 1986, p. 195). 
En el ámbito científico se suele emplear el término agresividad para hacer referencia a la tendencia o disposición inicial que da lugar a la posterior agresión. De hecho, Carrasco y González (2006) precisaron que la agresión constituye una conducta puntual, mientras que la agresividad consiste en una disposición o tendencia a comportarse agresivamente en distintas situaciones. De modo coincidente, Farnicka (2016) planteó que el constructo agresividad caracteriza la tendencia a comprometerse en el comportamiento agresivo en el futuro, siendo un rasgo de personalidad estable en el tiempo conducente al comportamiento incontrolado, agresivo y a la tendencia a externalizar los problemas.

Sin embargo, con el propósito de buscar mayores precisiones, emerge la necesidad de diferenciar el concepto de agresividad del concepto de impulsividad. Según Moeller, Barratt, Dougherty, Schmitz y Swann (2001), la impulsividad se relaciona con una predisposición a reaccionar rápidamente y de manera no planeada a estímulos internos o externos, sin reparo alguno por las consecuencias negativas que esta reacción pudiera tener para el individuo o para otros. Riaño-Hernández, Guillen y Buela-Casal (2015), haciendo una profunda revisión sistemática de la conceptualización de la impulsividad, concluyeron que es un constructo complejo que involucra características como toma de decisiones apresuradas, falta de atención, dificultad en el procesamiento de información e inhibición de la conducta.

La impulsividad es concebida como un atributo psicológico negativo e indeseable para la vida en comunidad (Pueyo, 2004). Riaño-Hernández et al., (2015) identificaron que este concepto presenta relaciones con otros constructos como: problemas de conducta, dificultades cognitivas, agresión y riesgo suicida. Para estos autores, las personas impulsivas tienden a un tipo de repuesta prematuro, sin demasiado análisis, que se genera cuando enfrentan problemas con varias opciones de solución, pero siendo solo una de ellas la correcta. Así, la impulsividad se relacionaría con déficit en los procesos cognitivos de evaluación y consideración de alternativas. De manera complementaria, para De la Peña-Fernández (2010), las dificultades se observan en el plano de la resolución de problemas y la toma de decisiones, en la demora de la gratificación y, subsidiariamente, en la ausencia de una perspectiva temporal a largo plazo que les facilitaría atender más convenientemente a las consecuencias de sus conductas.

\section{Antecedentes}

En el ámbito latinoamericano, Juárez, Dueñas y Méndez (2006), tomaron una muestra de 120 participantes de la Escuela de Policía Nacional de Boyacá, Colombia. Encontraron que el incremento en el tiempo de permanencia, rango e ingresos se encuentra vinculado al patrón violento. Mercadillo y Barrios (2011) plantearon un diseño etnográfico y psicométrico de tipo longitudinal en una muestra de 8 policías del departamento de Nezahualcóyotl, México, que les permitió conjeturar que el sistema neurocognitivo encargado de regular las reacciones inhibitorias y la impulsividad está fuertemente influido por los códigos morales y de comportamiento aprendidos durante el entrenamiento y la práctica policial. Rueda, Pinto y Ávila-Batista (2016), con una muestra de 178 integrantes de la policía militar del estado de Minas Gerais, Brasil, se basaron en las facetas de los cinco factores de la personalidad y encontraron que la inestabilidad emocional, la responsabilidad y la extraversión están estrechamente relacionadas con la impulsividad. 


\section{Objetivos e hipótesis}

Este trabajo se orienta, en general, a indagar los niveles de impulsividad y agresividad en los alumnos de Instituto Superior de Seguridad Pública. Más concretamente, pretende comprobar si existen diferencias de media en torno a estas variables según sexo y según año de formación, así como describir posibles relaciones entre las variables en estudio. Por tanto, se proponen las siguientes hipótesis:

H1: Los hombres presentarán diferencias de media significativas en términos estadísticos respecto de las mujeres en las variables en estudio.

H2: Los alumnos de tercer año presentarán niveles de impulsividad y agresividad más elevados que los alumnos de segundo año.

H3: Los cadetes con mayores niveles de impulsividad tenderán a ser más agresivos.

\section{Método}

\section{Diseño}

La presente investigación se enmarca en un nivel descriptivo, optándose para el análisis de los resultados un diseño de tipo descriptivo y correlacional.

\section{Participantes}

La extracción de la muestra estuvo condicionada por las limitaciones que el órgano directivo del Instituto Superior de Seguridad Pública dispuso para efectuar esta investigación. Concretamente, se trabajó con una muestra intencional, no probabilística, conformada por 58 cadetes de policía (39 varones y 19 mujeres), $M=41.32$ años y $D T=6.07$.

\section{Instrumentos de medición}

Cuestionario de Agresividad (AQ) adaptado en Argentina por Reyna, Lello-Ivacevich, Sanchez y Brussino (2011). Este instrumento ha sido diseñado originalmente por Buss y Perry (1992) para recoger información sobre los aspectos conductuales, cognitivos y emocionales asociados con la agresividad. Por tal razón, se ha utilizado ampliamente en estudios acerca del comportamiento agresivo y ha sido traducido a diferentes idiomas. Es un cuestionario que consta de 29 ítems, los cuales han de responderse siguiendo una escala tipo Likert de cinco puntos. Estos ítems se estructuran con base en cuatro subescalas:

Agresión Física: es definida como un ataque contra un organismo perpetrado por partes del cuerpo (brazos, piernas, dientes) o por el empleo de armas (cuchillo, cachiporra, revolver).

Agresión verbal: se trataría de la reacción verbal que descarga estímulos nocivos contra un organismo (repudio, crítica, derogación, insulto y amenaza).

Hostilidad: actitud que comporta una reacción verbal implícita de sentimientos negativos (mala disposición) y evaluaciones negativas de las personas y acontecimientos.

Ira: constituye una respuesta emocional caracterizada por una activación fisiológica y una expresión facial propia acompañada por sentimientos de enfado o enojo. 
Las dos primeras subescalas constituyen el componente instrumental de la agresividad, es decir el elemento proactivo con sus dos posibles formas de manifestación. La hostilidad conformaría el componente cognitivo a través del cual se activan los procesos intencionales de atribución de significados en tanto que la ira alude al componente emocional o afectivo que impulsa ciertas conductas y sentimientos. Se obtuvo, para esta versión, un coeficiente de confiabilidad de $\alpha=.88$.

Escala de Impulsividad de Barratt (Barratt Impulsiveness Scale, BIS-11), que ha sido adaptada y sometida a comprobación de validez en población carcelaria argentina por Folino et al., 2006. La undécima versión de la Escala de impulsividad de Barratt (BIS-11) es uno de los instrumentos de autorreporte más ampliamente utilizados para la valoración de la impulsividad. Consta de 30 ítems agrupados en torno a tres subescalas:

Impulsividad Cognitiva: implica la tendencia a actuar sin pensar, dejándose llevar por el ímpetu del momento.

Impulsividad Motora: refiere a la propensión a tomar decisiones rápidas, sin considerar las consecuencias negativas que tales decisiones tienen para sí mismo u otros.

Impulsividad no planificada: se caracteriza precisamente por la tendencia a no trazar planes, mostrando un mayor interés por el presente que por el futuro.

Además, la escala ofrece una puntuación total (Impulsividad general), que consiste en la suma de los valores correspondientes a las subescalas anteriormente mencionadas. Las opciones de respuestas se puntúan siguiendo una escala tipo Likert que va de 0 a 4 puntos y ha obtenido un índice de consistencia interna de $\alpha=.68$ (Folino et al., 2006).

Procedimiento

Inicialmente, se contó con la autorización formal de los responsables del Instituto Superior de Seguridad Pública. Luego, los cuestionarios fueron administrados de manera simultánea, tomando los recaudos necesarios para asegurar el anonimato, la confidencialidad de los formularios y el cumplimiento de todas las normas éticas. Se explicó verbalmente el alcance y propósito de esta investigación, temas que fueron reforzados por la lectura y posterior firma del consentimiento informado que formalizó los cuatro principios de la Bioética: no maleficencia (compromiso de no divulgar las respuestas brindadas ni actuar causando daño físico, mental y/o moral a los participantes del estudio); justicia (se garantiza a los integrantes de la muestra condiciones de igualdad, consideración y respeto antes, durante y después de responder los cuestionarios); beneficencia (se ofreció un taller de habilidades en regulación emocional una vez concluida la recolección de datos); y autonomía (se respetó la decisión de participar, manteniéndose la confidencialidad absoluta).

Una vez concluida la etapa de recolección de datos, estos fueron cargados y procesados en el programa estadístico SPSS versión 21 para Windows. Se obtuvo el análisis de los estadísticos descriptivos con la finalidad de observar la distribución de las variables en estudio. Tanto para comprobar la existencia de diferencias de media como para establecer la existencia de asociación entre las variables, se aplicaron pruebas no paramétricas en función del tamaño muestral obtenido. 


\section{Resultados}

Como se puede observar en la tabla 1, teniendo en cuenta los puntajes transformados, los valores más elevados están relacionados con la agresión verbal y hostilidad, en tanto que la variable agresión física presenta niveles sensiblemente menores. Respecto a las subescalas que componen la Escala de impulsividad de Barratt, se observa que los valores son indicativos de bajos niveles, particularmente en Impulsividad no planeada.

En la tabla 2 se aprecia que las mujeres han obtenido puntajes de media que son significativamente mayores respecto a los hombres en la variable hostilidad, lo que reflejaría una mayor tendencia a experimentar sentimientos negativos y a evaluar negativamente a las personas y acontecimientos. En la variable agresión física, los varones han presentado niveles más elevados respecto a las mujeres, lo que indicaría al sexo masculino como más proclive a emplear el aspecto instrumental asociado a la agresividad.

Es posible visualizar, en la tabla 3, diferencias de media altamente significativas entre la muestra de cadetes de segundo y tercer año. En las variables expresión emocional abierta, impulsividad motora, agresión verbal e ira se han encontrado valores más elevados en aquellos cadetes que cursan el tercer año.

Tabla 1

Estadísticos descriptivos para las variables en estudio $(\mathrm{N}=58)$

\begin{tabular}{|c|c|c|c|c|c|c|}
\hline & & $M$ & $D T$ & Asimetría & Curtosis & $\begin{array}{c}\text { Puntajes } \\
\text { transformados }\end{array}$ \\
\hline \multirow{4}{*}{ 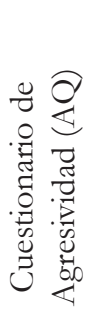 } & Agresión física & 16.17 & 4.20 & .938 & .994 & 44.91 \\
\hline & $\begin{array}{l}\text { Agresión } \\
\text { verbal }\end{array}$ & 11.96 & 3.30 & -.100 & -.956 & 59.80 \\
\hline & Ira & 14.17 & 4.25 & .592 & -.267 & 50.60 \\
\hline & Hostilidad & 18.44 & 4.75 & -.125 & -.556 & 57.62 \\
\hline \multirow{4}{*}{ 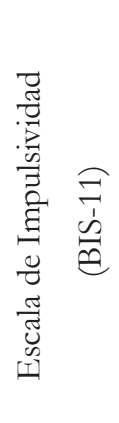 } & $\begin{array}{l}\text { Impulsividad } \\
\text { motora }\end{array}$ & 8.65 & 4.97 & .645 & .535 & 28.83 \\
\hline & $\begin{array}{l}\text { Impulsividad } \\
\text { cognitiva }\end{array}$ & 12.33 & 3.34 & -.182 & -.691 & 36.04 \\
\hline & $\begin{array}{l}\text { Impulsividad } \\
\text { no planeada }\end{array}$ & 13.79 & 4.53 & .557 & .235 & 24.02 \\
\hline & $\begin{array}{l}\text { Impulsividad } \\
\text { general }\end{array}$ & 34.77 & 9.86 & .148 & -.619 & 38.63 \\
\hline
\end{tabular}


Tabla 2

Diferencias de media y niveles de significación estadística encontrados en las variables en estudio respecto al sexo

\begin{tabular}{ccccccccc} 
& $\begin{array}{c}\text { Mujeres } \\
(N=19)\end{array}$ & $\begin{array}{c}\text { Hombres } \\
(N=39)\end{array}$ & $\begin{array}{c}\text { Dif. } \\
\text { media }\end{array}$ & & Prueba de Mann-Whitney \\
& $M$ & $D T$ & $M$ & $D T$ & & U Whitney & $Z$ & Sig. Bilat. \\
\hline $\begin{array}{c}\text { Agresión } \\
\text { Física }\end{array}$ & 14.50 & 3.01 & 16.92 & 4.46 & -2.41 & 538.0 & -1.96 & .042 \\
Hostilidad & 20.31 & 4.54 & 17.61 & 4.66 & 2.70 & 550.5 & 1.94 & .039 \\
\hline
\end{tabular}

Lo anterior podría estar sugiriendo los efectos que la instrucción policial tiene en la conformación de la subjetividad de sus miembros. Sin embargo, cabe destacar que la confirmación de esta sospecha debería ser saldada con un diseño de investigación exigente en el que se pueda comparar las puntuaciones en las variables dependientes medidas entre un grupo de control y grupos experimentales.

Como es factible observar en la tabla 4, se encontró una asociación negativa de baja intensidad entre agresión física y edad. Esto sugiere que los niveles de este tipo de agresión tienden a disminuir a medida que aumentaba la edad de las personas evaluadas. Por otra parte, se ha encontrado correlaciones positivas de moderada intensidad y altamente significativas a nivel estadístico entre la variable agresión física y las variables impulsividad general, impulsividad motora, impulsividad cognitiva e impulsividad no planeada.

Un panorama similar lo presenta la variable impulsividad motora, pues presenta, además, asociaciones positivas de baja a moderada intensidad con las variables agresión verbal, ira y hostilidad.

\section{Discusión y Conclusiones}

A pesar de que el acervo de investigaciones realizadas en el ámbito policial de Argentina, es aún escaso e incipiente, se ha encontrado en este trabajo evidencia que permite arribar a algunas conclusiones, dejando abierto el interés por estudiar este tipo particular de población. De acuerdo con los resultados obtenidos, se observan, en comparación con la población carcelaria Argentina, niveles de impulsividad significativamente más bajos en las tres dimensiones evaluadas (Folino et al., 2006).

Se hallaron puntuaciones media, de las subescalas impulsividad no planeada e impulsividad motora, similares en a las obtenidas en la adaptación y validación de la Escala de Impulsividad de Barratt en población hispana (Oquendo et al., 2001). No obstante, en la 
Tabla 3

Diferencias de media y niveles de significación estadística encontrados en las variables en estudio respecto al Año de cursada

\begin{tabular}{|c|c|c|c|c|c|c|c|c|}
\hline & \multicolumn{2}{|c|}{$\begin{array}{l}\text { Muestra de } \\
\text { Segundo año } \\
(N=30)\end{array}$} & \multicolumn{2}{|c|}{$\begin{array}{l}\text { Muestra de } \\
\text { Tercer año } \\
(N=28)\end{array}$} & \multirow[t]{2}{*}{$\begin{array}{l}\text { Dif. } \\
\text { media }\end{array}$} & \multicolumn{3}{|c|}{ Prueba de Mann-Whitney } \\
\hline & $M$ & $D T$ & $M$ & $D T$ & & $\begin{array}{c}\text { U } \\
\text { Whitney }\end{array}$ & $Z$ & Sig. Bilat. \\
\hline $\begin{array}{l}\text { Impulsividad } \\
\text { motora }\end{array}$ & 6.97 & 4.27 & 10.95 & 5.01 & -3.98 & 522.0 & -3.08 & .003 \\
\hline $\begin{array}{l}\text { Agresión } \\
\text { verbal }\end{array}$ & 11.03 & 2.96 & 13.23 & 3.39 & -2.19 & 589.5 & -2.48 & .017 \\
\hline Ira & 12.93 & 3.80 & 15.86 & 4.32 & -2.59 & 501.9 & -2.93 & .013 \\
\hline
\end{tabular}

Tabla 4

Coeficientes de correlación Rho de Spearman y significación bilateral entre las variables en estudio

\begin{tabular}{|c|c|c|c|c|c|c|}
\hline & & Edad & $\begin{array}{l}\text { Impulsividad } \\
\text { general }\end{array}$ & $\begin{array}{l}\text { Impulsividad } \\
\text { motora }\end{array}$ & $\begin{array}{l}\text { Impulsividad } \\
\text { cognitiva }\end{array}$ & $\begin{array}{l}\text { Impulsividad } \\
\text { no planeada }\end{array}$ \\
\hline \multirow{2}{*}{$\begin{array}{l}\text { Agresión } \\
\text { física }\end{array}$} & Coef. Corr. & $-.281 *$ & $.504^{* *} *$ & $.368^{* *}$ & $.450 * *$ & $.361 * *$ \\
\hline & Sig. bilat. & .043 & .000 & .007 & .001 & .009 \\
\hline \multirow{2}{*}{$\begin{array}{l}\text { Agresión } \\
\text { verbal }\end{array}$} & Coef. Corr. & & & $.278^{*}$ & & \\
\hline & Sig. bilat. & & & .046 & & \\
\hline \multirow{2}{*}{ Ira } & Coef. Corr. & & $.432 * *$ & $.490 * *$ & & \\
\hline & Sig. bilat. & & .001 & .000 & & \\
\hline \multirow{2}{*}{ Hostilidad } & Coef. Corr. & & $.345^{*}$ & $.333^{*}$ & & \\
\hline & Sig. bilat. & & .012 & .016 & & \\
\hline
\end{tabular}

$*=p<.05 . * *=p<.01$ 
subescala impulsividad cognitiva se obtuvieron valores sensiblemente superiores, lo cual sugiere que los policías tenderían a tomar decisiones apresuradas, precipitadas y poco meditadas (con una escasa o deficiente mediación cognitiva) ante la presencia de estímulos ambientales interpretados como amenazantes o provocadores. Lo anterior puede conducir a cometer un mayor número de errores en sus respuestas, sin prever las consecuencias negativas que estas puedan acarrear tanto para sí mismos como para los demás.

En este sentido, Barratt (1993) había sostenido que los individuos con puntuaciones elevadas en impulsividad cognitiva presentarían mayores dificultades en tareas que demanden atención durante periodos prolongados de tiempo. Si bien para el instrumento no se han establecido puntos de corte, Bobes-García et al. (2002) han propuesto considerar como referencia las medianas obtenidas en la validación española con pacientes psiquiátricos: Impulsividad cognitiva: 9.5; Impulsividad motora: 9.5; Impulsividad no planeada: 14; puntuación total: 32.5 . De acuerdo con esta perspectiva, la muestra en estudio presentaría en comparación niveles más bajos en Impulsividad cognitiva y valores mas elevados en Impulsividad no planeada, datos que resultan concordantes considerando la naturaleza de la muestra de referencia.

Respecto a los niveles de agresividad, se realizó una comparación con la muestra de estudiantes universitarios de Barcelona de la investigación realizada por Porras, Salamero y Sender (2002). Se obtuvieron, en la muestra local, valores de media más elevados en la subescala agresión física, puntuaciones inferiores en agresión verbal, así como puntajes equivalentes en las restantes dimensiones (Hostilidad e Ira). Esta particularidad podría ser entendida como la disposición preferencial por el uso potencial o real de la fuerza física. Este aspecto reviste especial importancia puesto que se trata de individuos próximos a graduarse de policía, quienes en su labor futura deberán observar el estado policial con su consecuente obligación a portar armas de fuego y con la capacidad de decidir, llegado el caso, sobre la vida y/o libertad de otras personas (Galvani, 2007).

En esta dirección, Saín (2004) afirmó que es en la "utilización del recurso de la coacción física, en donde se manifiesta la especificidad de la función policial” (p. 28). Además, Tudela-Poblete (2010) refirió que el empleo de la fuerza física constituye un rasgo definitorio de la actividad policial, marca su desempeño y su rúbrica idiosincrasia; por lo que confluiría en la construcción de una “... personalidad, estilo o carácter del trabajo policial, no como un fenómeno individual, sino como una matriz socialmente construida y transmitida mediante procesos de socialización” (p. 81).

Por otra parte, las puntuaciones de media inferiores en cuanto a la subescala agresión verbal podrían estar relacionadas con el hecho de que "en una institución jerarquizada como la policía, que glorifica el arte de la subordinación...” (Sirimarco, 2009, p. 74), los cadetes son condicionados para obedecer. De ahí que el discutir, cuestionar o desacatar las órdenes del superior son muestra de insolencia por parte del agente y pueden constituir motivo suficiente como para que este sea sumariado por falta disciplinaria (Galvani, 2007). Se debe señalar que los antecedentes aportados por la literatura científica, relacionados con el nivel de agresividad en policías, son muchas veces contradictorios y, posiblemente 
vinculados a cuestiones ambientales; así, Gonzáles-Fernández (2017) encontró valores promedios en la población policial de Lima-Perú, mientras que Rodríguez (2015) altos en agentes de Quito-Ecuador y Rojas-Solís y Morán (2015) bajos en policías de México.

En cuanto a la Hipótesis 1 (Los hombres presentarán diferencias de media significativas en términos estadísticos respecto de las mujeres en las variables en estudio), fue confirmada parcialmente, dada las diferencias de media halladas entre las variables. Se encontró que, mientras la muestra de hombres tiende a valores más elevados en agresión física, las mujeres lo hacen en la variable hostilidad, lo que sugiere que éstas recurrirían a formas más sutiles de agresión.

Estos resultados coinciden con los obtenidos en diversas investigaciones (AndreuRodríguez, et al., 2002; Porras, et al., 2002), en las que se afirma que tales diferencias son el reflejo de los valores sociales diferenciales aprendidos en relación con la expresión de la agresividad entre los géneros. Las mujeres serían socializadas para inhibir con mayor frecuencia las conductas agresivas directas, destacándose en ellas características como la sensibilidad, compasión y ternura (Eagly, Wood, \& Johannesen-Schmidt, 2004); mientras la demostración de valor, la inhibición afectiva, la tendencia a correr riesgo, la agresividad y dureza se presentan como conductas asociadas y vinculadas con la masculinidad (Catrillón, \& Vieco, 2002).

En efecto, Sirimarco (2009) resaltó que la agencia policial es, desde sus orígenes, una institución androcéntrica en donde se forja la configuración de un sujeto policial masculino, de manera que el cadete y posterior policía “... se piensa y representa como un sujeto superior, conquistador, dominante, y sobre todo, capaz de ser agente de la subordinación de otros" (p. 121).

Ahora bien, respecto de la Hipótesis 2 (Los alumnos de tercer año presentarán niveles de impulsividad y agresividad más elevados que los alumnos de segundo año), se encontraron valores de media más elevados en impulsividad motora, agresión verbal e ira en aquellos individuos que cursan el tercer año de la carrera Tecnicatura Superior en Seguridad Pública. En ese sentido, es posible dar cuenta de la presencia de acciones irreflexivas, inmediatas e incontroladas en respuesta a estímulos ambientales interpretados como amenazantes y en función del conjunto de reacciones emocionales, afectivas, faciales y verbales activadas por la circunstancia.

Lo anterior se exterioriza o comunica hacia otras personas vinculadas o asociadas al problema e incluye la descalificación (insulto) de estas, la crítica y la amenaza de un posible ataque. En otras palabras, estas puntuaciones reflejarían una mayor desregulación emocional, afectiva y conductual a medida que el sujeto transcurre el periodo final de formación en estas escuelas y se aproxima al ejercicio concreto de la labor policial, lo cual corrobora lo planteado por Mercadillo y Barrios (2011), quienes observaron en su investigación un incremento de impulsividad producto de códigos morales y de comportamientos aprendidos durante el entrenamiento.

A propósito, se ha de recordar que durante la cursada de este último año, los cadetes desempeñan prácticas en diferentes comisarías, tomando contacto directo con la realidad organizacional y laboral futura que habrán de desarrollar desde su rol profesional. Al 
respecto, Galvani (2007) señaló que la formación en estas escuelas se da en el marco de relaciones fuertemente autoritarias y de excesiva disciplina, en donde el contacto con la realidad laboral futura es tan solo parcial y centrada en los componentes normativos, procedimentales y estructurales que definen el "deber ser" policial. No obstante, el resto del aprendizaje (deber hacer) se da en el trabajo cotidiano y en el vínculo diario con funcionarios policiales más antiguos. En definitiva, ello probablemente explique las diferencias de media significativas halladas entre los alumnos de los respectivos cursos.

En lo referente a los índices de correlación obtenidos, los resultados señalarían que la variable edad se asocia únicamente y de manera negativa con la subescala agresión física. Por tanto, es posible señalar que la disposición preferencial por el empleo de la agresión física disminuye conforme avanza la edad de los sujetos. Ello es consistente con los hallazgos reportados por Archer (2004) y Andreu et al. (2002), quienes demostraron que la madurez adquirida con el paso del tiempo disminuye la tendencia a emitir conductas agresivas. Más precisamente, lo que estos autores señalan es que durante la edad adulta la expresión directa de la agresividad suele inhibirse (controlarse) y/o reemplazarse por medios indirectos y encubiertos de agresión.

Por otra parte, y de modo general, se observó que la totalidad de las variables de impulsividad presentaron asociaciones positivas con la variable agresión física. En tanto que la variable impulsividad motora manifestó una relación positiva con las variables agresión física, agresión verbal, ira y hostilidad. Por lo anterior, se puede afirmar que la Hipótesis 3 (Los cadetes con mayores niveles de impulsividad tenderán a ser más agresivos), ha sido confirmada.

Se entiende que tal relación podría explicarse por un déficit relacionado con la capacidad de reflexionar respecto de la situación a enfrentar (identificar la situación, definir sus causas, planificar en función del objetivo a proponerse). Al respecto, autores como Swann, Bjork, Moeller y Dougherty (2002) sostuvieron que la impulsividad es consecuencia de deficiencias cognitivas vinculadas al procesamiento de la información que conlleva a una inadecuada e incompleta evaluación del contexto, lo cual resulta indispensable a la hora de realizar una apropiada definición del problema y proponer estrategias de solución eficaces.

Desde esta perspectiva, la impulsividad es una respuesta rápida, no deseada y propensa a errores, que se produce ante la presencia de estímulos amenazantes y en ausencia del control cognitivo. Así, es probable que los cadetes que presentan un pobre control de sus impulsos tenderían a externalizar sus dificultades y/o frustraciones mediante acciones agresivas de ataque, destrucción o daño, sin reflexionar sobre los resultados probables que su propia conducta pueda tener tanto para si mismo como para los demás.

Este tipo de reacción se encuadraría en lo que diferentes autores coinciden en denominar como agresión reactiva-hostil-impulsiva y/o emocional. Esta consistiría en aquella conducta agresiva de naturaleza impulsiva, que viene precedida por la experiencia de ira y evaluaciones negativas respecto de las otras personas y cuya intención es ocasionar daño a estas o aquellos objetos valorados por el sujeto (Andreu-Rodríguez, 2009; Andreu, Ramírez, \& Raine, 2006; Palmero, \& Fernández-Abascal, 2002).

Los resultados encontrados en el presente estudio permiten reflexionar acerca de las emociones, creencias, valores y conductas que desde las instancias de formación, 
socialización y enculturación inicial se transmiten, animan, toleran, justifican e incluso recompensan; las que pueden descuidar aspectos como la prudencia, la prevención y el uso de todos los medios necesarios para evitar la violencia y lidiar con el estrés. Las peculiaridades del trabajo policial parecieran conducir hacia una representación de su función como natural e inevitablemente peligrosa, lo que lleva a aceptar el riesgo y el empleo de la violencia como parte intrínseca de su tarea.

Sin embargo, una mirada más abarcativa sobre la institución permitiría dar cuenta de que esa representación imposibilita la puesta en marcha de estrategias de prevención e intervención que apunten a prevenir el uso de la fuerza, el desgaste y la insatisfacción profesional, tanto en el ámbito individual, grupal y organizacional. En este sentido, conviene recordar a Shafir (2016), quien planteó que para frenar la agresión impulsiva y evitar proactivamente los trastornos de salud mental que suelen sufrir los oficiales de policía durante su formación, deben ser enseñadas habilidades de regulación emocional.

De modo concordante, Pereira-Basilio y Riccio (2017) han insistido que el cambio de enfoque en la formación de los oficiales de policía depende, en gran medida, de una capacitación efectiva que desplace la política de seguridad represiva. En ella, los oficiales de policía están entrenados para actuar de manera reactiva, por una política basada en la gestión y la prevención en la que los agentes son capacitados para reaccionar de manera proactiva en la resolución de los problemas que surgen en la vida cotidiana.

Finalmente, se señala que las limitaciones de este estudio estuvieron principalmente relacionadas con las dificultades para acceder a la muestra, así como los requisitos y condiciones impuestas para trabajar (control de los cuestionarios, tiempo mínimo de permanencia en la institución) debidas al hermetismo y resistencia presentados por el Instituto Superior de Seguridad. Tales condiciones preestablecidas imposibilitaron el acceso a una muestra conformada por un mayor número de cadetes, en la que participasen también los alumnos de primer año de la carrera. Por último, se sugiere, con base en lo que de este trabajo se desprende, la posibilidad de que las intervenciones psicológicas en el ámbito policial pudieran extenderse más allá del proceso de selección de los aspirantes a policía, convirtiéndose en un acompañamiento a lo largo de toda la carrera mediante una adecuada formación, continua supervisión y, en caso de ser necesario, con una asistencia técnica profesional.

\section{Referencias}

Alonso, J. (2008). Gobierno instituciones y tendencias de la formación policial en la República Argentina. Buenos Aires: Consejo de Seguridad Interior, Programa Naciones Unidas para el Desarrollo- PNUD.

Andreu, J. M., Peña, M. E., \& Graña, J. L. (2002). Adaptación psicométrica de la versión española del Cuestionario de Agresión. Psicothema, 14(2), 476-482.

Andreu, J. M., Ramírez, J. M., \& Raine, A. (2006). Un modelo dicotómico de la agresión: valoración mediante dos auto-informes (CAMA y RPQ). Psicopatología Clínica Legaly Forense, 6(1), 25-42. 
Andreu-Rodríguez, J. M. (2009). Propuesta de un modelo integrador de la agresividad impulsiva y premeditada en función de sus bases motivacionales y sociocognitivas. Psicopatología Clínica Legal y Forense, 98(1), 85-98.

Archer, J. (2004). Sex differences in aggression in real-world settings: A meta-analytic review. Review of General Psychology, 8(4), 291-322.

Barratt, E. (1993). Impulsivity: Integrating cognitive, behavioral, biological and environmental data. En McCown, W. G., Johnson, J. L., \& Shure, M. B. (eds., 1993). The impulsive client: Theory, research and treatment. Washington, DC: American Psychological Association.

Bobes-García, J, Portilla, M. P., Bascarán-Fernández, M. T., Sáiz-Martínez, P. A., \& Bousoño-García, M. (2002). Banco de instrumentos básicos para la práctica de la psiquiatría clínica. 2a Edición. Madrid: Editorial Ars Médica.

Buss, A. H., \& Perry, M. (1992). The aggression questionnaire. Journal of Personality and Social Psychology, 63(3), 452-459.

Carrasco, M. A., \& González, M. J. (2006). Aspectos conceptuales de la agresión: definición y modelos explicativos. Acción Psicológica 4(2), 7-38. doi: 10.5944/ap.4.2.478

Castrillón, D. M., \& Vieco, F. G. (2002). Actitudes justificativas del comportamiento agresivo y violento en estudiantes universitarios de la ciudad de Medellín Colombia. Revista de la Facultad Nacional de Salud Pública, 20(2), 51-66.

De la Peña-Fernández, M. E. (2010). Conducta Antisocial En Adolescentes: Factores de Riesgo y de Protección. Tesis Doctoral. Universidad Complutense de Madrid. Facultad de Psicología Departamento de Personalidad, Evaluación y Tratamiento Psicológico I.

Eagly, A. H., Wood, W., \& Johannesen-Schmidt, M. C. (2004). Social role theory of sex differences and similarities. In Eagly, A. H., Beall, A. E., \& Sternberg, R. J. (Eds). The psychology of gender. New York: Guilford Press.

Farnicka, M. 2016. Differences in Determinants of Indirect Perpetrator and Victim Behaviour. The Open Psychology Journal, 9(1), 149-159. doi: 10.2174/1874350101609010149

Folino, J. O., Escobar-Córdoba, F., \& Castillo J. L. (2006). Exploración de la validez de la Escala de Impulsividad de Barratt (BIS 11) en población carcelaria Argentina. Revista Colombiana de Psiquiatría, 35(2), 132-148.

Fromm, E. (1986). Anatomía de la destructividad humana. Madrid, España: Siglo XXI.

Galvani, M. (2007). La marca de la gorra: un análisis de la Policía Federal. Buenos Aires, Argentina: Capital Intelectual.

Gonzáles-Fernández, A. Y. (2017). Sindrome de Burnouty agresividad en los efectivos de la Policía Nacional del Perú de las comisarías del distrito de Comas, Lima. Tesis de Licenciatura, Facultad de Humanidades, Escuela Profesional de Psicología, Universidad 
César Vallejo. Recuperado de http://repositorio.ucv.edu.pe/bitstream/ handle/UCV/3267/Gonzales_FAY.pdf? sequence $=1$ \&isAllowed $=\mathrm{y}$

Juárez, F., Dueñas, A. N., \& Méndez, Y. (2006). Patrones de comportamiento violento en la Policía Nacional de Colombia. International Journal of Clinical and Health Psychology, 6(1), 127-143.

Mercadillo, R. E., \& Barrios, F. A. (2011). Brain Correlates of Impulsivity in Police Officers: A Neurocognitive and Ethnological Exploration. The Open Criminology Journal, 4(2) 54-60.

Moeller, F. G, Barratt, E. S., Dougherty, D. M., Schmitz, J. M., \& Swann, C. A. (2001). Psychiatric aspects of impulsivity. American Journal of Psychiatry, 158(11), 17831793. doi: 10.1176/appi.ajp.158.11.1783

Oquendo, M. A, Baca-García, E., Graver, R., Morales, M., Montalván V., \& Mann, J. J. (2001). Spanish adaptation of the Barratt Impulsiveness Scale (BIS-11). The European Journal of psychiatry, 15(3), 147-155.

Palmero, F., \& Fernández-Abascal, E. G. (2002). Emociones básicas II (ira, tristeza y asco). En Palmero, F., Fernández-Abascal, E. G., Martínez, F., \& Cholis, M. (2002). Psicología de la motivación y la emoción. Madrid, España: McGraw-Hill.

Pereira-Basilio, M., \& Riccio, V. (2017). The Challenge of Military Police Training in the State of Rio de Janeiro: Utopia or Real Possibility? Universal Journal of Management, 5(12) 570-580. doi: 10.13189/ujm.2017.051204

Porras, S., Salamero, M., \& Sender, R. (2002). Adaptación española del Buss y Perry Aggression Questionnaire. Cuadernos de Medicina Psicosomática y Psiquiatría de Enlace, 60(61), 7-12.

Pueyo, A. A. (2004). Evaluación de la impulsividad y riesgo en el uso de armas de fuego en policías y fuerzas de seguridad. Revista Catalana de Segurelat Pública, 14, 63-78.

Reyna, C., Sánchez, A., Lello, M., \& Brussino, S. (2011). The Buss-Perry Aggression Questionnaire: Construct validity and gender invariance among Argentinean adolescents. International Journal of Psychological Research, 4(2), 30-37. doi: $10.21500 / 20112084.775$

Riaño-Hernández, D., Guillén, A., \& Buela-Casal, G. (2015). Conceptualización y evaluación de la impulsividad en adolescentes: una revisión sistemática. Universitas Psychologica, 14(3), 1077-1090. doi: 10.11144/Javeriana.upsy14-3.ceia

Rodríguez, B. (2015). Estilos comunicacionalesy niveles de agresividad en los agentes de la jefatura provincial de Pichincha de la Dirección Nacional de la Policía Especializada en Niños, Niñas y Adolescentes. Tesis de Licenciatura. Facultad de Ciencias Psicologícas, Universidad Central del Ecuador. Recuperado de http:/ /www.dspace.uce.edu.ec/handle/25000/7056

Rojas-Solís, J. L., \& Morán, T. (2015). Síndrome de Burnout y agresividad en policías mexicanos: Resultados preliminares de un estudio de casos exploratorio. Revista Internacional PEI: Por la Psicología y Educación Integral, 9(5), 189-203. 
Rueda, F.J., Pinto, L. P., \& Ávila-Batista, A. C. (2016). Impulsividad y facetas dela personalidad: relación entre instrumentos de medida. Ciencias Psicológicas, 10(1), 7-16.

Saín, M. (2004). Política, policía y delito. La Red Bonaerense. Buenos Aires: Capital Intelectual.

Shafir, T. (2016). Using movement to regulate emotion: Neurophysiological findings and their application in psychotherapy. Frontiers in Psychology, 7, 1451. doi: 10.3389/ fpsyg.2016.01451

Sirimarco, M. (2009). De civil a policía: una etnografía del proceso de incorporación a la institución policial. Buenos Aires, Argentina: Teseo.

Swann, A. C, Bjork, J. M., Moeller, F. G., \& Dougherty, D. M. (2002). Two models of impulsivity: relationship to personality trait and psychopathology. Biological Psychiatric, 51(12), 988-994. doi: 10.1016/S0006-3223(01)01357-9

Tudela-Poblete, P. (2010). Cultura Policial y control interno. Revista Estudios Policiales, (7), 74-104. 